\title{
Evaluation of Single-Step Trans-Epithelial Photorefractive Keratectomy Postoperative Corneal Haze with Oculus Corneal Densitometry
}

\author{
Anas Adel ${ }^{\text {a }}$, Yousry Fekry ${ }^{\text {b }}$, Mona G. Sharawy ${ }^{\text {b }}$, Haitham M. Fayek ${ }^{\text {b }}$ \\ ${ }^{a}$ Department of Ophthalmology, \\ Abstract: \\ Helwan University, Egypt. \\ ${ }^{\mathrm{b}}$ Department of Ophthalmology, \\ Benha faculty of medicine, \\ Benha University, Egypt. \\ Aim: to evaluate post-operative corneal haze trans-epithelial \\ photorefractive keratectomy and ensure significance of the corneal \\ densitometry software as an objective tool for routine follow up. \\ Correspondence to: Anas \\ Adel, Department of \\ Ophthalmology, Helwan \\ University, Egypt. \\ Methods: Trans-epithelial photorefractive keratectomy using the \\ Amaris 1050RS excimer laser (SCHWIND eye-tech-solutions \\ GmbH, Kleinostheim, Germany) with contralateral eye control \\ through conventional alchohol assisted photorefractive keratectomy \\ Email: \\ to 44 eyes in a 22 patients with post-operative 6-month follow-up. \\ anasadelalmassry@gmail.com \\ Assessing corneal haze objectively through imaging with the \\ Received: 3June 2020 \\ Pentacam ® HR (Oculus Optikgeräte $\mathrm{GmbH}$ ) corneal densitometry \\ Accepted: 23 June 2020 \\ setting with subjective clinical examination to ensure sensitivity and \\ specificity. Results: comparable clinical haze grading with faster \\ haze resolution in the t-PRK after 3 month $(\mathrm{P}=0.48)$. Objective \\ haze assessment showed no significance, but with data parameters explaining the clinical haze. \\ Epithelial healing after 3 days was $90.9 \%$ in t-PRK and 59.1\% in c-PRK (P=0.015). The dryness \\ assessment was comparable $(\mathrm{P}=0.191)$. Conclusion: comparable results between the two groups \\ with faster haze and visual recovery in t-PRK. Corneal densitometry proved a good tool for \\ following up corneal haze along with the clinical assessment expressing good correlation.
}

Key words: Trans-epithelial Photorefractive Keratectomy, corneal haze, corneal densitometry

\section{List of Abbreviations:}

1.t-PRK: trans-epithelial photorefractive keratectomy

2.c-PRK: conventional photorefractive keratectomy

3.GSU: greyscale unit 



\section{Introduction}

Assessing the corneal haze is a corner stone in evaluating the post-operative course of surface ablation procedures. As a result, establishing an objective corneal haze assessment tool would be of great value for patients follow up, studying corneal healing \& evaluation of procedures post-operatively.

The lack of such objective available diagnostic device, in spite of the various previous studies conducted to do so, was the motivating force for this study, especially with insufficient data in literature regarding the efficiency of the corneal densitometry setting of the Pentacam $®$ HR (Oculus Optikgeräte $\mathrm{GmbH}$ ), to act as such a tool. ${ }^{[1-}$ 6]

Literature shows corneal densitometry studies in keratoconus, Fuch's dystrophy, normative data studies and studies postsurgeries. ${ }^{[7-16]}$

This study concentrated on the postoperative photorefractive keratectomy haze comparing contralateral trans-epithelial photorefractive keratectomy (t-PRK) and conventional alcohol assisted photorefractive keratectomy (c-PRK) and assessing the objective specificity and sensitivity of the corneal densitometry setting to do so.
Pentacam system is a non-invasive system that outputs numerical values, thereby allowing objective assessment. The corneal densitometry software provides a standardized method of selective quantification of corneal opacities.

Maximum and average opacities can be shown in selected layers. Corneal opacities are viewed for defined annuli and depths, facilitating uniform evaluation. This function served previously as a basis in the normative data studies. ${ }^{[12-16-17-]}$

\section{Patient and method}

The study was carried out in accordance with the tenets of the Declaration of Helsinki. A written informed consent was taken from all patients prior to surgery.

The study was concluded under the supervision of the ophthalmology department staff in the college of medicine Benha University in the period between September 2018 and February 2020.

The study was designed as a prospective interventional study of 22 subjects ( 22 eyes) with contralateral eye control (22 eyes, total of 44 treatments). As a matched control group, eliminating individual variations potentially affecting the outcome, plus the 
advantage of having the same patient to subjectively compare \& evaluate the treatment modalities between eyes.

For every patient, the choice of eye treatments were randomized \& the implementation of intervention or control, with the fellow eye receiving the alternate treatment.

Patients \& observers were masked to the treatment modalities done to each eye.

Inclusion criteria included bilateral treatment to fit the design of the study, age over 18, simple or compound myopic refraction (astigmatism not exceeding 3D), spherical equivalent between -1D up to -7D, stable refraction for 18 months preceding surgery, a planned corneal stromal ablation maximum of $110 \mu \mathrm{m}$, higher order aberrations $(\mathrm{HOA})<0.50$ um OU \& CDVA of 0.6 Snellen decimal or better.

Eyes with corneal epithelial pathology (dystrophies or scarring), keratoconus, ocular inflammation, eyelids or conjunctival infections, glaucoma, cataract, posterior segment pathologies, previous surgical ocular history, positive systemic medical history, or other contraindications for refractive surgery were excluded from the study. All patients received similar bilateral treatment by the same surgeon at the same session.

Soft contact lenses were seized 1 week prior to imaging \& examination for surgery, \& 3 weeks in case of hard contact lenses.

The preoperative assessment was done according to the full standard routine ophthalmological examination, corneal tomography with the Pentacam ${ }^{\circledR}$ and with the COMBI WAVEFRONT ANALYZER including both functions of the SCHWIND SIRIUS (SCHWIND eye-tech-solutions $\mathrm{GmbH}$ Kleinostheim, Germany) tomography imaging \& ocular aberrometer function of the SCHWIND PERAMIS (SCHWIND eye-tech-solutions $\mathrm{GmbH}$, Kleinostheim, Germany).

Both analyzed at $3 \mathrm{~mm} \& 6 \mathrm{~mm}$ diameter. Pentacam ${ }^{\circledR}$ corneal densitometry uses an internal standard corneal densitometry analysis (figure 1) software measures the backscattered light over a 12-mm diameter corneal area. The Pentacam software (Version 1.21r65) automatically calculated all parameters. 


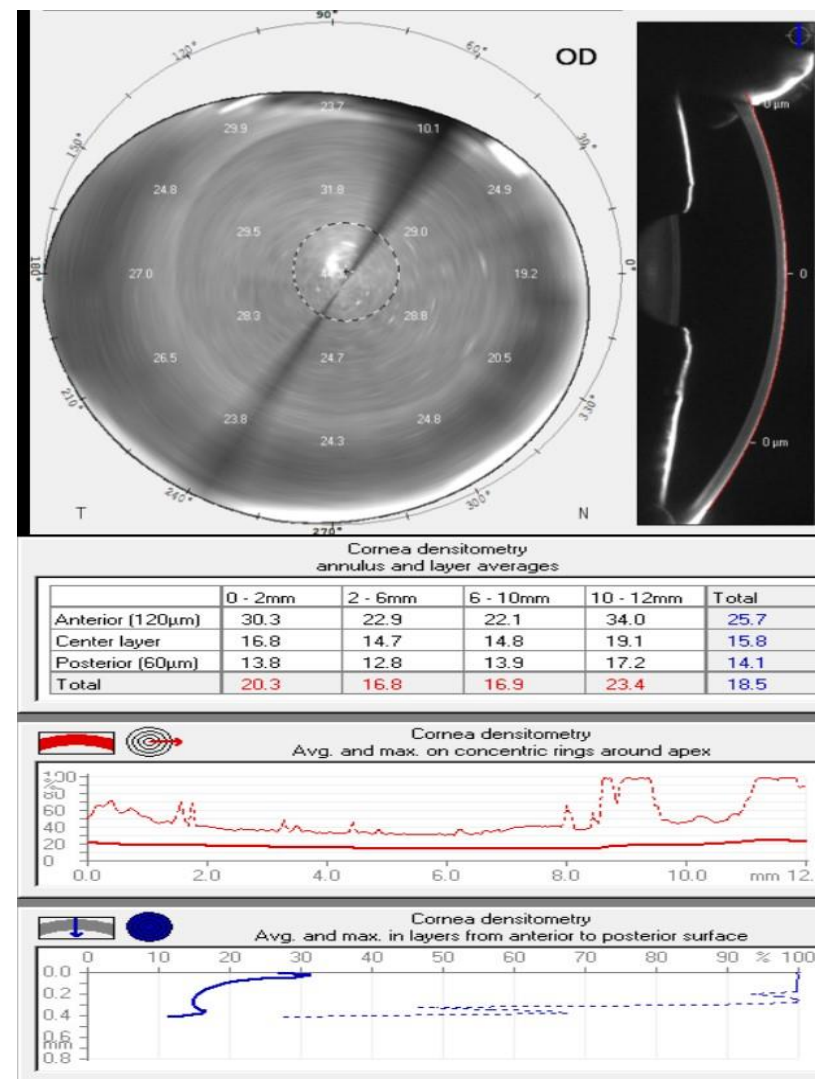

\section{Surgical technique}

Calculation of total surgical time from speculum insertion to removal during procedure, was done

Both eye groups treatment were with the Amaris 1050RS excimer laser (SCHWIND eye-tech-solutions $\mathrm{GmbH}$, Kleinostheim, Germany) with $1050 \mathrm{~Hz}$ pulse rate, ablation of 1.3 seconds per diopter and a $7 \mathrm{D}$ eye tracker to compensate static \& dynamic cyclotorsion (SCC and DCC).

In the c-PRK group, treatment was with the standard PRK protocol. Epithelial removal was done with a $20 \%$ ethanol, applied using an $8-9 \mathrm{~mm}$ well according to the patient's data interpretation by the surgeon $\&$ the determined ablation zone accordingly, followed by epithelial delamination with a spatula.

In the t-PRK group, the excimer laser administration was in a single continuous session to ablate both the epithelium \& stroma in a single step. Both groups, ablation profiles were aspheric aberration neutral (non-wave front-guided treatment). According to a population-based epitheliumthickness profile ${ }^{[18-]}$, the ablation plan utilized $55 \mu \mathrm{m}$ centrally \& $65 \mu \mathrm{m}$ peripherally with an approximately $8 \mathrm{~mm}$ treatment zone and $6.3 \mathrm{~mm}$ optical zone.

For all eyes in both groups, the patients received a standardized intraoperative application of mitomycin-C (MMC) $0.02 \%$ for 8-12 seconds, immediately after excimer ablation. Then, the eye was irrigated by copious amounts of chilled balanced saline solution (BSS).

At the end of laser ablation, a high-oxygencontent silicone hydrogel soft contact lens was placed over the cornea with a drop of topical steroid (prednisolone 1\%) \& topical antibiotic (gatifloxacin 0.5\%) instilled.

\section{Postoperative care and follow up}

The regimen was consistent between the two groups, the patients was treated with topical instillation of antibiotic drops (gatifloxacin 
$0.5 \%$ ) six times daily for one-week, topical instillation of steroid drops (prednisolone $1 \%$ ) six times daily (tapered over 5-weeks) $\&$ artificial tear drops (sodium hyaluronate $0.2 \%$ ) six times daily for 3-months. An adjuvant therapy was added to the standard treatment regimen to augment the epithelial healing process; vitamin C $500 \mathrm{mg}$ CAPS twice per day \& vitamin A 50.000 I.U. CAPS once per day both for 2 weeks.

Postoperative assessment $\&$ follow-ups were performed on day 3,1 week, 1, 3 and 6 months after surgery. Both, patients \& the observer were masked to the assignment of eyes. In the first postoperative follow up contact lens removal, or replacement, and re-epithelization was noted and measured. At each subsequent visit, we recorded postoperative corneal haze at 1 week, 1, 3, 6, months clinically grading its degree with the Slit-Lamp Bio-microscope. Postoperative imaging with the Pentacam $®$ was taken in the 1, 3, \& 6 months follow-ups in order to record the corneal densitometry as an unbiased objective method of assessing corneal haze. Other parameters recorded included delayed epithelial healing assessed with sterile fluorescein staining, dryness with fluorescein assisted break up time and tear film analyzer in the 6 month follow up with the SCHWIND SIRIUS.

\section{Haze assessment:}

Haze grading subjectively measured through clinical grading according to Fantes et al ${ }^{[19-]}$ and Fahd et al ${ }^{[20-]}$. The grading was accordingly $0=$ no haze, $+0.5=$ trace haze on oblique illumination; $+1=$ corneal cloudiness not interfering with the visibility of fine iris details; $+2=$ mild interference with fine iris details; +3 and $+4=$ details of the iris are unclear. Objective assessment was through the Pentacam $®$ corneal densitometry setting measuring the degree of haze through the postoperative stromal corneal density changes.

\section{Statistical method:}

The statistical package for the Social Sciences (SPSS) version 25 (IBM Corp., Armonk, NY, USA) was used for data coding and entry. Summarization of data using mean, standard deviation, median, minimum and maximum in quantitative data and using frequency (count) and relative frequency (percentage) for categorical data. The non-parametric Mann-Whitney test (Chan, 2003a) was used for Comparisons between quantitative variables. Chi-square $(\chi 2)$ test was performed comparing categorical data. Exact test was used instead when the expected frequency is less than 5 (Chan, 2003b). P-values less than 0.05 were considered as statistically significant. ${ }^{[21-22-]}$ 


\section{Results}

\section{Surgical parameters:}

The mean optical zone was $6.37 \pm 0.20$ in the study group and $6.32 \pm 0.13$ in the contralateral control group $(\mathrm{P}=0.602)$. The mean treatment surgical time for the study group was $6.89 \pm 1.81$ and $10.34 \pm 3.23$ in the contralateral control group showing lesser operative time in t-PRK with high significance $(\mathrm{P}=<0.001)$. The postoperative data results (table 2), shows the epithelial healing time in the t-PRK group compared to the c-PRK group in the 3 days postoperative $1^{\text {st }}$ follow up showed $90.9 \%$ complete epithelial healing in the study group compared to only $59.1 \%$ in the contralateral control group with a statistical significance of $(\mathrm{P}=0.015)$. The dryness assessment with the fluorescein assisted break up time clinically in $1 \& 3$ months follow-ups and the non-invasive tear film (NIF) break up time with the tear film analyzer of the SCHWIND SIRIUS in the 6month follow up showed no statistical significance between the two study groups. $(\mathrm{P}=0.191)$. The clinical haze grading (table 3.) was comparable between the 2 groups with in all follow ups, but with a slight significance in the 3 months follow up in favor for haze resolution in the t-PRK group $(P=0.48)$. The objective haze assessment with the Pentacam ${ }^{\circledR}$ (table 4.) showed no statistical significance in all follow-ups.

Table 1. preoperative demographic and clinical data

\begin{tabular}{lccc}
\hline Parameter & \multicolumn{1}{c}{ t-PRK } & c-PRK & P Value \\
\hline Treatments (n) & 22 & 22 \\
Females (n) & 16 & 16 \\
Males (n) & 6 & 6 & \\
Age (y) & $29.48 \pm 6.21$ & \\
& \multicolumn{2}{c}{ Mean \pm SD } \\
UDVA (d) & $0.25 \pm 0.14$ & $0.26 \pm 0.14$ & 0.854 \\
CDVA (d) & $0.97 \pm 0.15$ & $0.98 \pm 0.16$ & 0.837 \\
Sphere (D) & $-2.28 \pm 1.44$ & $-2.34 \pm 1.24$ & 0.925 \\
Cylinder (D) & $-1.26 \pm 0.67$ & $-1.11 \pm 0.71$ & 0.454 \\
SE (D) & $-2.91 \pm 1.43$ & $-2.90 \pm 1.28$ & 0.963 \\
\hline
\end{tabular}

$\mathrm{c}-\mathrm{PRK}=$ conventional photorefractive keratectomy; $\mathrm{t}-\mathrm{PRK}=$ trans-epithelial photorefractive keratectomy UDVA = uncorrected distance visual acuity; CDVA = corrected distance visual acuity; $\mathrm{SE}=$ spherical equivalent; $\mathrm{d}=$ decimal; $\mathrm{D}=$ Diopters . 
Table 2. Post-operative results.

\begin{tabular}{llll}
\hline \multicolumn{2}{c}{ Mean \pm SD } & \\
Parameter & t-PRK & c-PRK & P Value \\
\hline Epi. (\%) & $90.9 \%$ & $59.1 \%$ & 0.015 \\
B.U.T. $<$ 5 sec. (\%) & & & 0.664 \\
1 months & $9.1 \%$ & $18.2 \%$ & 0.724 \\
3 months & $41.2 \%$ & $35.3 \%$ & 0.191 \\
6 months & $8.89 \pm$ & $11.19 \pm 5.08$ & \\
\hline
\end{tabular}

c-PRK = conventional photorefractive keratectomy; t-PRK = trans-epithelial photorefractive keratectomy; .Epi.\%: epithelial healing percentage; BUT: break up time ; sec.: seconds

Table 3. Post-operative clinical haze grading

\begin{tabular}{|c|c|c|c|}
\hline parameter & $\begin{array}{l}\text { t-PRK } \\
\text { Mean } \pm \text { SD }\end{array}$ & c-PRK & $\begin{array}{l}\mathbf{P} \\
\text { Value }\end{array}$ \\
\hline \multicolumn{4}{|c|}{ Haze post 1 week } \\
\hline 0.5 & $80.0 \%$ & $52.4 \%$ & \\
\hline 1 & $20.0 \%$ & $38.1 \%$ & \\
\hline 2 & $0.0 \%$ & $9.5 \%$ & 0.125 \\
\hline \multicolumn{4}{|c|}{ Haze post 1 month } \\
\hline clear & $18.2 \%$ & $9.1 \%$ & \\
\hline 0.5 & $81.8 \%$ & $81.8 \%$ & \\
\hline 1 & $0.0 \%$ & $9.1 \%$ & 0.419 \\
\hline \multicolumn{4}{|c|}{ Haze post 3 month } \\
\hline clear & $57.9 \%$ & $31.6 \%$ & \\
\hline 0.5 & $31.6 \%$ & $68.4 \%$ & 0.049 \\
\hline 1 & $10.5 \%$ & $0.0 \%$ & \\
\hline \multicolumn{4}{|c|}{ Haze post 6 month } \\
\hline clear & $85.0 \%$ & $70.0 \%$ & \\
\hline 0.5 & $15.0 \%$ & $30.0 \%$ & 0.451 \\
\hline
\end{tabular}

c-PRK = conventional photorefractive keratectomy; t-PRK = trans-epithelial photorefractive keratectomy. 
Table 4. Post-op. corneal densitometry average

\begin{tabular}{llll}
\hline & \multicolumn{1}{l}{ Mean \pm SD } & \\
parameter & t-PRK & PRK & P Value \\
\hline 1 month GSU & & \\
Ant. 0-2 mm & $27.19 \pm 2.53$ & $29.22 \pm 6.30$ & 0.481 \\
Ant. 2-6mm & $22.56 \pm 1.53$ & $24.92 \pm 5.60$ & 0.094 \\
Ant. 6-10mm & $24.18 \pm 3.34$ & $25.30 \pm 4.95$ & 0.589 \\
3 month GSU & & & 0.603 \\
Ant. 0-2 mm & $25.15 \pm 1.59$ & $25.74 \pm 2.16$ & 0.271 \\
Ant. 2-6mm & $21.98 \pm 1.49$ & $22.77 \pm 1.90$ & 0.644 \\
Ant. 6-10mm & $22.05 \pm 5.36$ & $23.49 \pm 3.13$ & \\
6 months GSU & & & 0.445 \\
Ant. 0-2 mm & $24.49 \pm 1.47$ & $24.99 \pm 1.82$ & 0.862 \\
Ant. 2-6mm & $21.81 \pm 1.41$ & $21.97 \pm 1.65$ & 0.461 \\
Ant. 6-10mm & $23.78 \pm 3.37$ & $23.32 \pm 3.76$ & \\
\hline
\end{tabular}

c-PRK = conventional photorefractive keratectomy; t-PRK = trans-epithelial photorefractive keratectomy; Ant.: anterior; GSU: greyscale unit

\section{Discussion:}

Our study showed a comparable visual outcome safety and efficacy in both study groups, but with lesser overall surgical time in the t-PRK, which is on agreement to t-PRK earlier studies by Fadlallah et al. ${ }^{[23-]}$.

Our results also showed a rather significant delayed epithelial healing in the alcoholassisted group, which might be explained by the possible ethanol toxic effect to the epithelial layer. Furthermore, the longer exposure time with subsequent dehydration of the stromal bed during the procedure, which is reflected by a mean treatment surgical time for the study group of $6.89 \pm 1.81$ and $10.34 \pm$ 3.23 in the contralateral control group showing lesser operative time in t-PRK with high significance $(\mathrm{P}=<0.001)$. Other studies [24-25-23], concluded a roughly equal results of faster re-epithelialization time with transepithelial removal. 
The corneal haze grading subjectively clinically or objectively by the corneal densitometry were non-conclusive statistically in which, coincides with the previous studies results. Densitometry results were comparable to ours, in which the 3-month postoperative value was $37.81 \mathrm{GSU}$, and the 12 month one was 26.92 GSU. ${ }^{[14-]}$ Somewhat lesser numbers were reported by other researchers. [1516-]

The differential variation in numerical results could be attributable to inconsistent examination protocols and personal variation in data acquisition, especially with different software versions and calculation method.

Nevertheless, clinical haze grading proved with statistical evident a more rapid haze recovery in the t-PRK group after 3 months of surgery in regard to the alcohol assisted group $(\mathrm{P}=0.49)$. Equality reported in achieving a clear cornea in the 6 months follow up.

Moreover, the densitometry showed a linear relation between subjective haze grading, maximum corneal densitometry in the anterior $0-2 \mathrm{~mm}$ zone and the anterior $2-6$ zone with good predictability.

\section{Conclusion}

Despite the drawback of having lesser number of eyes participating in the study, but the importance of data collection from the same patient, eliminating any individual, social variation or treatment incompliance that may affect the result outcome was of more far value to a better study result. Also, insuring patient compliance, to study follow-ups through better monitoring and careful preoperative patient selection, is seeked.

As previously mentioned our study covers a gap in literature data regarding densitometry haze assessment specifically in post-operative photorefractive keratectomy. Despite the lack of data in the previous studies conducted regarding the subject most studies conquer, that the corneal densitometry would be a good tool for objective haze assessment, side by side to clinical haze grading in post PRK patients' follow-ups.

\section{References}

1. Andrade HA, McDonald MB, Liu JC, Abdelmegeed M, Varnell R, Sunderland G.et al. Evaluation of an opacity lensometer for determining corneal clarity following excimer laser photoablation. Refract Corneal Surg 1 990; 6: 34 6-5 J.

2. Lohmann CP, Timberlake GT, FitzkeFW, Gartry DS, Muir MK, Marshall J.et al. Corneal light scattering after excimer laser photorefractive keratectomy: the objective measurements of haze. Refract Corneal Surg 1992; 8: 114-2J. 
3. Essepian JP, Rajpal RK, Azar DT, et al. The use of confocal microscopy in evaluating corneal wound healing afier excimer laser keratectomy. Scanning Microscopy J994;16:300-4.

4. High frequency ultrasound quantitative grading of postexcimer cornea scarring. Invest Ophthalmol Vis Sci 1993; 34:S802.

5. Braunstein RE, Jain S, McCally RL, Stark WJ, Connolly PJ, Azar DT.et al. Objective measurement of" corneal light scattering after excimer laser kertectomy. Ophthalmology 1996; 1 03:439-43.

6. Jain S, Khoury JM, Chamon W, Azar DT.et al. Corneal light scattering after laser in situ keratomileusis and photo refractive keratectomy. Am J Ophthalmol 199 5; J 20: 532-4.

7. Sorcha Ni Dhubhghaill, Jos J. Rozema, Marie-Jose Tassignon; et al. Corneal Scheimpflug Densitometry Values measured by Pentacam in Fuchs Endothelial Dystrophy, ARVO 2014, Program Number: 2447 Poster Board Number: B0232.

8. Alnawaiseh M, Rosentreter A, Prokosch V, Eveslage M, Eter N, Zumhagen L. et al. Changes in corneal densitometry in patients with fuchs endothelial dystrophy after endothelial keratoplasty. Curr Eye Res $2016 ; 1-5$.

9. Lopes B, Ramos I, Ambrosio R Jr. et al. Corneal densitometry in keratoconus. Cornea 2014; 33: 1282 1286.

10. Bhatt UK, Fares U, Rahman I, Said DG, Maharajan SV, Dua HS.et al. Outcomes of deep anterior lamellar keratoplasty following successful and failed 'big bubble'. Br J Ophthalmol 2012; 96: 564-569.
11. Pircher N, Pachala M, Prager F, Pieh S, Schmidinger G.et al. Changes in straylight and densitometry values after corneal collagen crosslinking. J Cataract Refract Surg 2015; 41: 1038-1043.

12. Ni Dhubhghaill S, Rozema JJ, Jongenelen S, Ruiz Hidalgo I, Zakaria N, Tassignon MJ. et al. Normative values for corneal densitometry analysis by Scheimpflug optical assessment. Invest Ophthalmol Vis Sci 2014; 55: 162-168.

13. Cennamo G, Forte R, Aufiero B, La Rana A.et al. Computerized Scheimpflug densitometry as a measure of corneal optical density after excimer laser refractive surgery in myopic eyes. J Cataract Refract Surg 2011; 37: 1502-1506.

14. Poyales, F., Garzón, N., Mendicute, J. et al. Corneal densitometry after photorefractive keratectomy, laser-assisted in situ keratomileusis, and small-incision lenticule extraction. Eye 31, 1647-1654 (2017).

15. Fares U, Otri AM, Al-Aqaba MA, Faraj L, Dua HS.et al. Wavefront-optimized excimer laser in situ keratomileusis for myopia and myopic astigmatism: refractive outcomes and corneal densitometry. J Cataract Refract Surg 2012; 38: 2131-2138.

16. Sorcha Ni Dhubhghaill, Jos J. Rozema, Sien Jongenelen, Irene Ruiz Hidalgo, Nadia Zakaria,MarieJose Tassignon; et al. Normative Values for Corneal Densitometry Analysis by Scheimpflug Optical Assessment, IOVS, January 2014, Vol. 55, No. 1, 164.

17. https://www.pentacam.com/int/ophthalmologistdiagnostic-without-pentacam/models/pentacamrhr/optional-software.html. 
18. Sin S, Simpson TL. The repeatability of corneal and corneal epithelial thickness measurements using optical coherence tomography. Optom Vis Sci. 2006; 83:360-365.

19. Fantes FE, Hanna KD, Waring GO 3rd, Pouliquen Y, Thompson KP, Savoldelli M. et al. Wound healing after excimer laser keratomileusis (photorefractive keratectomy) in monkeys. Arch Ophthalmology. 1990 May; 108(5):665-75.

20. Fahd D dJ, Jain S, Azar D. Corneal haze after refractive surgery. In: Ali_o J, Azar DT, eds, et al. Management of Complications in Refractive Surgery. Berlin, German, Springer, 2008; 179-186.

21. Chan YH (2003a): Biostatistics102: Quantitative Data - Parametric \& Non-parametric Tests. Singapore Med J.; 44(8): 391-396.
22. Chan YH (2003b): Biostatistics 103: Qualitative Data -Tests of Independence. Singapore Med J.; 44(10): 498-503.

23. Fadlallah A, Fahed D, Khalil K, Dunia I, Menassa J, El Rami H, et al. Transepithelial photorefractive keratectomy: Clinical results. J Cataract Refract Surg. 2011; 37:1852-1857.

24. Zarei-Ghanavati S, Shandiz JH, Abrishami M, Karimpour M. et al. Comparison of mechanical debridement and trans-epithelial myopic photorefractive keratectomy: A contralateral eye study. J Curr Ophthalmol. 2019; 31(2):135-141. Published 2019 Jan 23. Doi:10.1016/j.joco.2019.01.003

25. Naderi M, Jadidi K, Mosavi SA, Daneshi SA. et al. Transepithelial Photorefractive Keratectomy for Low to Moderate Myopia in Comparison with Conventional Photorefractive Keratectomy. Journal of Ophthalmic \& Vision Research. 2016 Oct-Dec; 11(4):358-362. DOI: 10.4103/2008-322X.19407.

To cite this article: Anas Adel, Yousry Fekry, Mona G. Sharawy, Haitham M. Fayek. Evaluation of Single-Step Trans-Epithelial Photorefractive Keratectomy Postoperative Corneal Haze with Oculus Corneal Densitometry. BMFJ 2020; 37 (surgical issue): 55-65. DOI: 10.21608/bmfj.2020.31762.1273 